\title{
Iron status and risk factors of iron deficiency among pregnant women in Singapore: a cross-sectional study
}

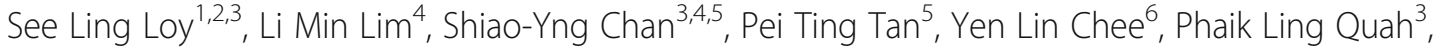
Jerry Kok Yen Chan ${ }^{1,2}$, Kok Hian Tan ${ }^{2,7}$, Fabian Yap ${ }^{2,8,9}$, Keith M. Godfrey ${ }^{10,11}$, Lynette Pei-Chi Shek ${ }^{3,12,13}$, Mary Foong-Fong Chong ${ }^{3,14}$, Michael S. Kramer ${ }^{4,15,16}$, Yap-Seng Chong ${ }^{3,4,5}$ and Claudia Chi ${ }^{4,5^{*}}$

\begin{abstract}
Background: Iron deficiency is the most prevalent nutrient deficiency and the most common cause of anaemia worldwide. Because of the increased iron requirements during pregnancy, iron deficiency can lead to maternal anaemia and reduced newborn iron stores. We examined the proportion and risk factors of iron deficiency among pregnant women in a developed Asian country.
\end{abstract}

Methods: Within a prospective cohort in Singapore, 985 Asian women were assessed for iron status at 26-28 weeks' gestation, with plasma ferritin and soluble transferrin receptor (STfR) measurements. Iron status was determined according to plasma ferritin concentrations at $\geq 30 \mu \mathrm{g} / \mathrm{L}$ (iron sufficiency), 15 to $<30 \mu \mathrm{g} / \mathrm{L}$ (modest iron depletion) and $<15 \mu \mathrm{g} / \mathrm{L}$ (severe iron depletion). Multivariable ordinal logistic regression was used to analyze risk factors for modest and severe iron depletion.

Results: The median (25-75th percentile) plasma ferritin concentration was 24.2 (19.9-30.6) $\mu \mathrm{g} / \mathrm{L}$. Overall, 660 (67.0\%) and 67 (6.8\%) women had modest and severe iron depletion, respectively. Higher plasma sTfR was observed in women with severe iron depletion than among those with iron sufficiency (median 17.6 versus 15.5 $\mathrm{nmol} / \mathrm{L} ; p<0.001)$. Age < 25 years (odds ratio 2.36 ; $95 \%$ confidence interval $1.15-4.84$ ), Malay $(2.05 ; 1.30-3.24)$ and Indian (1.98; 1.14-3.44) ethnicities (versus Chinese), university qualification $(1.64 ; 1.13-2.38)$, multiparity (1.73; $1.23-2.44)$ and lack of iron-containing supplementation $(3.37 ; 1.25-8.53)$ were associated with increased odds of modest and severe iron depletion.

Conclusions: Nearly three-quarters of Singaporean women were iron deficient in the early third trimester of pregnancy. These results suggest universal screening and supplementation of at-risk pregnancies may be evaluated as a preventive strategy.

Trial registration: NCT01174875. Registered 1 July 2010 (retrospectively registered).

Keywords: Anaemia, Ferritin, Iron status, Pregnancy, Risk factor, Singapore, Soluble transferrin receptor

\footnotetext{
* Correspondence: claudia_chi@nuhs.edu.sg

${ }^{4}$ Department of Obstetrics \& Gynaecology, National University Hospital, 5

Lower Kent Ridge Road, Singapore 119074, Singapore

${ }^{5}$ Department of Obstetrics \& Gynaecology, Yong Loo Lin School of Medicine,

National University of Singapore, National University Health System, 1E Kent

Ridge Road, Singapore 119228, Singapore

Full list of author information is available at the end of the article
}

(c) The Author(s). 2019 Open Access This article is distributed under the terms of the Creative Commons Attribution 4.0 International License (http://creativecommons.org/licenses/by/4.0/), which permits unrestricted use, distribution, and reproduction in any medium, provided you give appropriate credit to the original author(s) and the source, provide a link to the Creative Commons license, and indicate if changes were made. The Creative Commons Public Domain Dedication waiver (http://creativecommons.org/publicdomain/zero/1.0/) applies to the data made available in this article, unless otherwise stated. 


\section{Background}

Iron deficiency is the most prevalent global nutrient deficiency and the most common cause of anaemia worldwide [1, 2]. Iron deficiency represents a spectrum ranging from iron depletion without anaemia (reduced iron stores with a normal haemoglobin $(\mathrm{Hb})$ concentration) to eventual overt anaemia, where the iron supply is insufficient to maintain a normal $\mathrm{Hb}$ concentration [3]. Pregnant women are particularly vulnerable to iron deficiency due to substantial increase of iron requirement during pregnancy to support the expansion of erythrocyte mass and plasma volume, and foetal-placental growth [1, 4]. The World Health Organization (WHO) estimates that at least $30-40 \%$ of pregnant women are iron deficient and that nearly half are anaemic [5].

For pregnancy, the European Food Safety Authority [6] and the UK Committee on Medical Aspects of Food Policy [7] recommend no increase in iron intake over that for non-pregnant women. The extra iron requirements during pregnancy are considered to be met through cessation of menstrual losses, increased intestinal absorption and mobilisation of maternal iron stores [8]. However, a large proportion of pre-pregnant women or those of reproductive age have low iron stores $[9,10]$, predisposing them to an increased risk of iron deficiency when becoming pregnant [8]. In Singapore, a developed country, one in five non-pregnant women of reproductive age were found to be anaemic [11].

Either anaemic or non-anaemic iron deficiency prior to and during pregnancy can have adverse consequences for both the mother and offspring, especially with respect to neonatal iron-deficient condition $[5,12]$. It was previously thought that neonate was protected from iron deficiency as the developing fetus could acquire sufficient iron from the mother even when she was iron deficient [13]. However, it is now documented that neonatal iron stores can be compromised when the mother is iron deficient or anaemic [13]. Studies on rhesus macaques suggest that infants born to iron deficient mothers before pregnancy had low iron stores after birth [14]. In humans too, newborns from iron deficient mothers at delivery were found to have low iron stores, indicating there is a limited capacity for the fetus to accumulate iron from mothers with low stores [15]. Identifying risk factors for maternal iron deficiency may therefore be helpful in developing preventive strategies to improve offspring health.

Multiple biomarkers have been used to measure iron status. Of these, plasma (or serum) ferritin is the most clinically applicable in pregnancy and has been proposed as the most sensitive single screening test for iron stores [3]. The plasma ferritin threshold used to define iron deficiency in pregnancy varies [16]. According to the World Health Organization [5], plasma ferritin levels of lower than $15 \mu \mathrm{g} / \mathrm{L}$ indicate iron depletion (loss of iron stores) at all stages of pregnancy [3]. This threshold also confirms the presence of iron deficiency anaemia [17]. The Committee for Standards in Haematology from United Kingdom recommends iron supplementation in pregnancy for plasma ferritin less than $30 \mu \mathrm{g} / \mathrm{L}$ [3], a threshold also widely used in clinical practice, including in Singapore, to guide therapy for iron deficiency in pregnancy [16]. This threshold has higher specificity than the lower threshold or when compared with other pregnancy biomarkers [18].

Although iron deficiency anaemia in pregnancy has been a frequent focus of research, few studies have investigated iron deficiency per se in pregnancy [10], leading to uncertainty about its clinical and public health significance. This is particularly true for women living in relatively affluent settings. In this study in highly developed Singapore, we aimed 1) to examine the proportion of iron deficiency in women during the early third trimester of pregnancy, and 2) to assess risk factors associated with iron deficiency in pregnancy.

\section{Methods}

\section{Study design and participants}

Data were drawn from the Growing Up in Singapore Towards healthy Outcomes (GUSTO) pregnancy cohort study (www.clinicaltrials.gov, NCT01174875), detailed elsewhere [19]. This study was conducted according to the guidelines laid down in the Declaration of Helsinki. Ethical approval was obtained from the Domain Specific Review Board of Singapore National Healthcare Group (reference D/09/021) and the Centralised Institutional Review Board of SingHealth (reference 2009/280/D).

Pregnant women attending antenatal visits $(<14$ weeks' gestation) in KK Women's and Children's Hospital $(\mathrm{KKH})$ and National University Hospital (NUH) were recruited into the GUSTO study between June 2009 and September 2010. KKH and NUH are the two major public maternity units in Singapore. Recruited women were Singapore citizens or permanent residents between 18 and 50 years of age with biparentally homogeneous ethnicity (Chinese, Malay or Indian). Those who conceived naturally were included in this study. Women receiving chemotherapy or psychotropic drugs and those with type 1 diabetes mellitus were excluded. Informed written consent was obtained from all women prior to recruitment.

\section{Data collection}

Recruited women returned to the hospitals at 26-28 weeks' gestation for a follow-up study visit. Detailed interviews were conducted in the clinics by trained staff. Data on maternal socio-demographics, educational attainment, obstetric history, smoking status, 
iron-containing supplementation and anaemia history were collected. Women were asked about the highest level of education attained. Number of previous pregnancies and their outcomes were recorded to determine parity, which included all live- and stillbirths occurring at or after 24 weeks' gestation, to classify women as nulliparous or parous. Positive smoking status was defined as any cigarette smoking in the current pregnancy. Data on iron-containing supplements, including those taken as part of a multivitamin and mineral supplement or prenatal supplement, was recorded if it was taken for more than once a week in the current pregnancy. Women were asked if they had any history of anaemia in previous pregnancies, either antenatally or post-partum. Data on maternal $\mathrm{Hb}$ concentration $(\mathrm{g} / \mathrm{dl})$ in early pregnancy (14 weeks' gestation or less) was collected from the hospital medical records. Women were classified as anaemic if their $\mathrm{Hb}$ was less than $11 \mathrm{~g} / \mathrm{dL}$ [5].

\section{Anthropometry}

Self-reported pre-pregnancy weight and measured booking weight at the first antenatal clinic visit $(\leq 14$ weeks' gestation) were recorded. Height was measured with a portable stadiometer (Seca 213, Hamburg, Germany) at 26-28 weeks gestation. Body mass index (BMI) was determined using the formula of weight $(\mathrm{kg}) /$ height $\left(\mathrm{m}^{2}\right)$. Since the early pregnancy BMI obtained at the first clinic visit was strongly correlated with self-reported pre-pregnancy BMI $(\mathrm{r}=0.96, p<0.001)$, was free from recall bias and had a lower percentage of missing values than pre-pregnancy BMI (5.7\% vs. $7.7 \%)$, it was used for all study analyses. BMI status was categorized as $<23$ versus $\geq 23 \mathrm{~kg} / \mathrm{m}^{2}$ based on cut-off points for Asian populations [20].

\section{Plasma ferritin and soluble transferrin receptor assessments} At 26-28 weeks' gestation, maternal fasting blood samples were collected for the measurements of plasma ferritin and soluble transferrin receptor (sTfR). Plasma ferritin $(\mu \mathrm{g} / \mathrm{L})$ was measured using the sandwich enzyme-linked immunosorbent assay (ELISA) method (AssayMax Human Ferritin ELISA, AssayPro, United States) with an intra-assay coefficient of variation $(\mathrm{CV})$ of $2.9 \%$. The kit standard (AssayMax Human Ferritin Standard) was used as a control, which has been calibrated against WHO International Standard. Women were classified as having iron sufficiency, modest iron depletion and severe iron depletion based on plasma ferritin concentrations of $\geq 30,15$ to $<30$ and $<15 \mu \mathrm{g} / \mathrm{L}$, respectively $[3,5]$. Both modest and severe iron depletion were defined as iron deficiency. Since ferritin is an acute phase protein whose concentration can increase markedly during infection and other inflammatory conditions [16], we quantified the levels of sTfR as an additional biomarker of iron deficiency, since it is considered to be less affected by acute-phase reactants $[3,21]$. Elevated sTfR indicates the presence of functional iron deficiency. Plasma sTfR (nmol/L) was measured using an ELISA (Human sTFr ELISA, BioVendor, Czech Republic) with an intra- and inter-assay CV of 10.9 and 4.8\%, respectively. Control human serum samples (BioVendor Quality Control) were run in each assay as an internal control.

\section{Statistical analysis}

Descriptive statistics are presented as percentages for categorical variables; means, standard deviations, medians and 25-75th percentiles for continuous variables. Comparisons of demographics and characteristics between women with iron sufficiency, iron depletion and severe iron depletion were performed using Fisher's exact tests for categorical variables, ANOVA or Kruskal-Wallis tests for continuous variables. Spearman correlation was used to analyse the continuous association between maternal $\mathrm{Hb}$ in early pregnancy and plasma ferritin at 26-28 weeks' gestation.

Ordinal logistic regression with three ordinal levels was performed for multivariable analyses to assess independent risk factors for iron depletion and severe iron depletion. Compared to a series of binary logistic regression or using multinomial logistic regression, the use of an ordinal logistic regression model helps to increase the power by making full use of the structure of an ordinal scale, producing a more stable estimate and summary with a broad interpretation, applicable across multiple dichotomizations of outcome [22]. In determining variables to be included or excluded from the multivariable model, it has been shown that methods using pre-determined $p$-value criteria in the univariate analysis are inappropriate, and likewise for automated variable selection procedures (e.g. forward, stepwise) [23]. This is because confounding effects and inter-correlations between independent variables are not being considered, which can lead to biased and distorted outcomes [23]. A better way to determine which variables should be included in the multivariable model is by using clinical judgement [23], as done in other studies identifying risk factors of an outcome $[24,25]$. In this analysis, we selected the potential risk factors and built the model based on a literature review [26-28], clinical knowledge and by using a directed acyclic graph. In multivariable ordinal logistic regression analysis, we entered the following potential risk factors simultaneously into the model: maternal age $(<25,25-34$ or $\geq 35$ years $)$, BMI $\left(<23\right.$ or $\left.\geq 23 \mathrm{~kg} / \mathrm{m}^{2}\right)$, ethnicity (Chinese, Malay or Indian), education (below or at university levels), parity (nulliparous or multiparous), smoking status 
(no or yes), iron-containing supplementation (no or yes) and history of anaemia (no or yes). The fit of model and proportional odds assumption were checked and met. The proportional odds ratio as presented in this study could be viewed as independent from the degree of severity used to classify the iron status and was thus, valid over all cut-points simultaneously.

Missing values for maternal BMI $(n=6)$, education $(n=13)$, parity $(n=1)$, smoking status $(n=2)$, iron-containing supplementation $(n=97)$ and history of anaemia $(n=1)$ were imputed 100 times using multiple imputation analyses by chained equations. The results of the 100 analyses were pooled using Rubin's rule. Complete-case analysis was performed as a sensitivity analysis $(n=871)$. All statistical analyses were two-sided with a 5\% significance level and were performed using SPSS software, Version 20 (USA).

\section{Results}

Of 1152 recruited pregnant women who conceived naturally, 1090 (94.6\%) of them returned for a study visit at 26-28 weeks' gestation. A total of 977 (89.6\%) women had sufficient plasma for analysis of sTfR concentrations; while 985 (90.4\%) women had sufficient plasma for analysis of ferritin concentrations and were included in the present analyses (Fig. 1). Compared to excluded women, those included were more likely to have age greater than
35 years $(21.9$ versus $13.1 \%$; $P<0.001)$ and attained university education (33.7 versus $22.8 \% ; P=0.003$ ). They were, however, similar in every other factor assessed, including BMI status, ethnicity, parity, smoking status, iron-containing supplementation and history of anaemia (see Additional file 1: Table S1).

Table 1 shows the demographics and characteristics of women categorized by iron status. Overall $(n=$ 985), $73.8 \%(n=727)$ of pregnant women were iron deficient (ferritin $<30 \mu \mathrm{g} / \mathrm{L}$ ). Among these women, the majority had modest iron depletion (ferritin 15 to $<30 \mu \mathrm{g} / \mathrm{L}, \quad n=660 ; 67.0 \%)$, while $6.8 \% \quad(n=67)$ had severe iron depletion (ferritin $<15 \mu \mathrm{g} / \mathrm{L}$ ). Compared to women with iron sufficiency (ferritin $\geq 30 \mu \mathrm{g} / \mathrm{L}$ ), those with modest iron depletion were more likely to belong to the Malay (27.6 versus $21.7 \% ; P<0.001$ ) or Indian (21.8 versus $13.6 \% ; P<0.001)$ ethnic groups, to attain university education ( 36.5 versus $29.5 \% ; P=0.043$ ), to be multiparous ( 60.0 versus $51.2 \% ; P=0.013$ ), less likely to have taken iron-containing supplements during pregnancy ( 85.5 versus 93.4\%; $P=0.001$ ) and had higher plasma sTfR concentration (median 16.7 versus 15.5 nmol/L; $P<0.001)$. Out of 422 pregnant women with available information on $\mathrm{Hb}$ in early pregnancy, 81 (19.2\%) had anaemia, $91 \%$ of whom had iron deficiency (modest and severe iron depletion) at 26-28 weeks. Among non-anaemic women in early pregnancy $(n=341), 255(74.8 \%)$ were iron deficient at

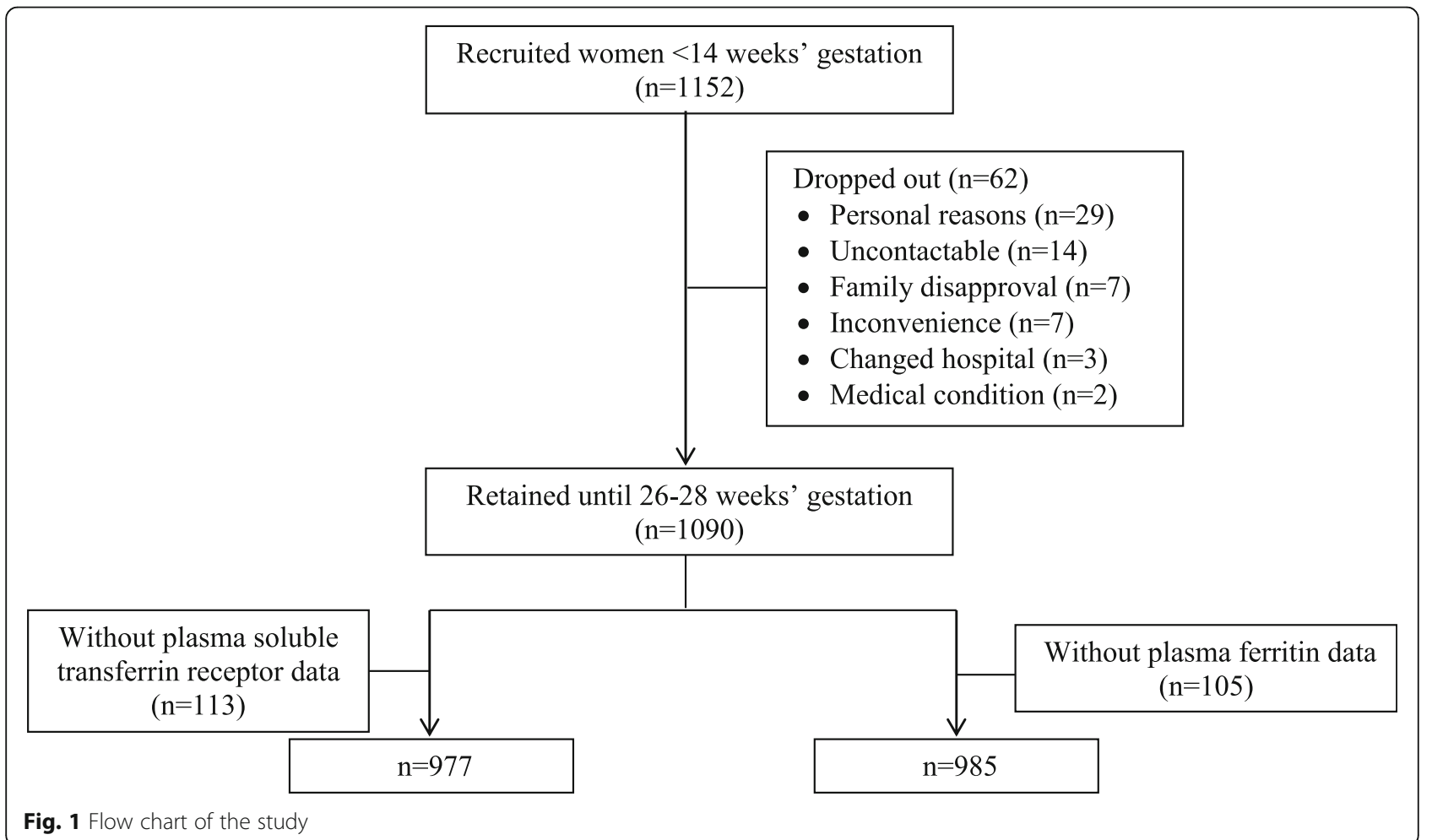


Table 1 Maternal characteristics by iron status based on plasma ferritin concentrations in the GUSTO study $(n=985)$

\begin{tabular}{|c|c|c|c|c|c|}
\hline Characteristics & $\begin{array}{l}\text { Total } \\
(n=985)\end{array}$ & $\begin{array}{l}\text { Iron sufficiency } \\
(n=258 ; 26.2 \%)\end{array}$ & $\begin{array}{l}\text { Modest iron depletion } \\
(n=660 ; 67.0 \%)\end{array}$ & $\begin{array}{l}\text { Severe iron depletion } \\
(n=67 ; 6.8 \%)\end{array}$ & $p^{a}$ \\
\hline Age, $\%$ & & & & & 0.044 \\
\hline$<25$ years & 12.8 & 10.1 & 12.9 & 22.4 & \\
\hline $25-34$ years & 65.3 & 64.0 & 66.2 & 61.2 & \\
\hline$\geq 35$ years & 21.9 & 26.0 & 20.9 & 16.4 & \\
\hline Body mass index, $\mathrm{kg} / \mathrm{m}^{2}$ & & & & & 0.410 \\
\hline Mean & 23.6 & 23.6 & 23.6 & 24.4 & \\
\hline Standard deviation & 4.8 & 4.7 & 4.7 & 5.2 & \\
\hline Body mass index, $\%$ & & & & & 0.727 \\
\hline$<23 \mathrm{~kg} / \mathrm{m}^{2}$ & 53.9 & 54.7 & 54.1 & 49.3 & \\
\hline$\geq 23 \mathrm{~kg} / \mathrm{m}^{2}$ & 46.1 & 45.3 & 45.9 & 50.7 & \\
\hline Ethnicity, \% & & & & & $<0.001$ \\
\hline Chinese & 54.4 & 64.7 & 50.6 & 52.2 & \\
\hline Malay & 26.6 & 21.7 & 27.6 & 35.8 & \\
\hline Indian & 19.0 & 13.6 & 21.8 & 12.0 & \\
\hline Education, \% & & & & & 0.016 \\
\hline None/Primary/Secondary & 66.3 & 70.5 & 63.5 & 77.6 & \\
\hline University & 33.7 & 29.5 & 36.5 & 22.4 & \\
\hline Parity, \% & & & & & 0.046 \\
\hline Nulliparous & 42.5 & 48.8 & 40.0 & 43.3 & \\
\hline Multiparous & 57.5 & 51.2 & 60.0 & 56.7 & \\
\hline Smoking status, \% & & & & & 0.751 \\
\hline No & 97.5 & 96.9 & 97.7 & 97.0 & \\
\hline Yes & 2.5 & 3.1 & 2.3 & 3.0 & \\
\hline Iron-containing supplementation, \% & & & & & 0.003 \\
\hline No & 12.5 & 6.6 & 14.5 & 14.9 & \\
\hline Yes & 87.5 & 93.4 & 85.5 & 85.1 & \\
\hline History of anaemia, \% & & & & & 0.952 \\
\hline No & 94.8 & 95.0 & 94.7 & 95.5 & \\
\hline Yes & 5.2 & 5.0 & 5.3 & 4.5 & \\
\hline Hemoglobin at early pregnancy $\mathrm{g} / \mathrm{dL}$ & & & & & 0.010 \\
\hline Median & 11.9 & 12.3 & 11.8 & 11.8 & \\
\hline Interquartile range & $11.2-12.6$ & $11.6-13.0$ & $11.0-12.6$ & $10.1-12.4$ & \\
\hline Anaemia at early pregnancy $\%$ & & & & & 0.005 \\
\hline No & 80.8 & 92.5 & 77.9 & 74.2 & \\
\hline Yes & 19.2 & 7.5 & 22.1 & 25.8 & \\
\hline Plasma ferritin, $\mu \mathrm{g} / \mathrm{L}$ & & & & & $<0.001$ \\
\hline Median & 24.2 & 36.2 & 22.6 & 12.3 & \\
\hline Interquartile range & $19.9-30.6$ & $32.3-42.1$ & $19.7-25.6$ & $9.4-13.9$ & \\
\hline Plasma soluble transferrin receptor, ${ }^{c} \mathrm{nmol} / \mathrm{L}$ & & & & & $<0.001$ \\
\hline Median & 16.4 & 15.5 & 16.7 & 17.6 & \\
\hline Interquartile range & $14.3-19.7$ & $13.6-18.2$ & $14.4-20.0$ & $15.0-21.6$ & \\
\hline
\end{tabular}

GUSTO Growing Up in Singapore Towards healthy Outcomes. Values are presented as percentages for categorical variables, and median (interquartile range) for continuous variables. Iron sufficiency, modest and severe iron depletion are defined as having plasma ferritin concentrations of $\geq 30 \mu \mathrm{g} / \mathrm{L}, 15$ to $<30 \mu \mathrm{g} / \mathrm{L}$ and $<15 \mu \mathrm{g} / \mathrm{L}$, respectively

${ }^{a} p$ values for differences between groups are obtained from Fisher's exact tests for categorical variables, ANOVA or Kruskal-Wallis tests for continuous variables

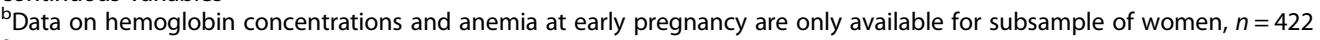

'Data on plasma soluble transferrin receptor are only available for 977 women 
26-28 weeks. A significant correlation was observed between $\mathrm{Hb}$ in early pregnancy and plasma ferritin at $26-28$ weeks' gestation $(r=0.22, p<0.001)$.

Table 2 shows the results of the univariable and multivariable ordinal logistic regression analyses. In the multivariable model, which was of good fit, maternal age $<25$ years (OR 2.36; 95\% CI 1.15, 4.84), Malay (OR 2.05; 95\% CI 1.30, 3.24) and Indian (OR 1.98; 95\% CI $1.14,3.44)$ ethnicities, university qualification (OR 1.64; 95\% CI 1.13, 2.38), multiparity (OR 1.73; 95\% CI 1.23, 2.44) and lack of iron-containing supplementation during pregnancy (OR 3.37; 95\% CI 1.25, 8.53) were associated with a significantly increased odds of modest and severe iron depletion. In the sensitivity analysis based on women with complete data $(n=871)$, the results remained similar (see Additional file 2: Table S2).

\section{Discussion}

During pregnancy, iron status tends to decline with advancing gestation, which can be due to iron mobilization or to haemodilution that peaks at 24-26 weeks [12]. Therefore, different cut-offs to define iron deficiency across gestation have been suggested [10]. Using stained bone marrow aspirates as the gold standard to evaluate iron status among pregnant women, most of those with an absence of stainable bone marrow haemosiderin iron have plasma ferritin $<30 \mu \mathrm{g} / \mathrm{L}$ in the second and third trimesters of pregnancy [18]. In the present study, at 26-28 weeks of gestation, only a quarter of pregnant women had sufficient iron stores, as defined by a plasma ferritin of at least $30 \mu \mathrm{g} / \mathrm{L}$. The majority of women (74\%) in our developed country was found to be iron deficient (ferritin $<30 \mu \mathrm{g} / \mathrm{L}$ ), in which $67 \%$ had modest iron

Table 2 Factors associated with iron status during pregnancy assessed by ordinal logistic regression analyses $(n=985)$

\begin{tabular}{|c|c|c|c|c|c|c|}
\hline \multirow[t]{2}{*}{ Characteristics } & \multicolumn{3}{|l|}{ Univariable } & \multicolumn{3}{|l|}{ Multivariable } \\
\hline & Ordinal $O^{a}$ & $95 \% \mathrm{Cl}$ & $P$ & Ordinal $O^{a}$ & $95 \% \mathrm{Cl}$ & $P$ \\
\hline \multicolumn{7}{|l|}{ Age } \\
\hline$<25$ years & 2.00 & $0.99,4.01$ & 0.050 & 2.36 & $1.15,4.84$ & 0.019 \\
\hline $25-34$ years & 1.31 & $0.93,1.86$ & 0.126 & 1.26 & $0.88,1.80$ & 0.209 \\
\hline$\geq 35$ years & Reference & & & Reference & & \\
\hline \multicolumn{7}{|l|}{ Body mass index } \\
\hline$<23 \mathrm{~kg} / \mathrm{m}^{2}$ & Reference & & & Reference & & \\
\hline$\geq 23 \mathrm{~kg} / \mathrm{m}^{2}$ & 1.04 & $0.75,1.44$ & 0.818 & 0.90 & $0.65,1.26$ & 0.551 \\
\hline \multicolumn{7}{|l|}{ Ethnicity } \\
\hline Chinese & Reference & & & Reference & & \\
\hline Malay & 1.83 & $1.17,2.85$ & 0.008 & 2.05 & $1.30,3.24$ & 0.002 \\
\hline Indian & 2.17 & $1.22,3.85$ & 0.008 & 1.98 & $1.14,3.44$ & 0.015 \\
\hline \multicolumn{7}{|l|}{ Education } \\
\hline None/ Primary/ Secondary & Reference & & & Reference & & \\
\hline University & 1.36 & $0.93,1.97$ & 0.110 & 1.64 & $1.13,2.38$ & 0.010 \\
\hline \multicolumn{7}{|l|}{ Parity } \\
\hline Nulliparous & Reference & & & Reference & & \\
\hline Multiparous & 1.48 & $1.07,2.05$ & 0.016 & 1.73 & $1.23,2.44$ & 0.002 \\
\hline \multicolumn{7}{|l|}{ Smoking status } \\
\hline No & Reference & & & Reference & & \\
\hline Yes & 0.73 & $0.32,1.65$ & 0.449 & 0.62 & $0.27,1.40$ & 0.250 \\
\hline \multicolumn{7}{|c|}{ Iron-containing supplementation } \\
\hline Yes & Reference & & & Reference & & \\
\hline No & 3.46 & $1.23,9.74$ & 0.019 & 3.37 & $1.25,8.53$ & 0.016 \\
\hline \multicolumn{7}{|l|}{ History of anemia } \\
\hline No & Reference & & & Reference & & \\
\hline Yes & 1.05 & $0.50,2.22$ & 0.900 & 0.95 & $0.44,2.04$ & 0.893 \\
\hline
\end{tabular}


depletion (ferritin 15 to $<30 \mu \mathrm{g} / \mathrm{L}$ ) and $7 \%$ had severe iron depletion (ferritin $<15 \mu \mathrm{g} / \mathrm{L}$ ). Women with age $<25$ years, Malay or Indian ethnicity, attained university qualification, multiparity and those not using iron-containing supplements during pregnancy were more likely to be iron depleted.

The prevalence of iron deficiency in pregnant women varies worldwide [28-31], depending on the definition used, study population, gestational age at assessment and assay method. Based on a plasma ferritin threshold of $<30 \mu \mathrm{g} / \mathrm{L}$, the proportion of iron deficiency among women at $26-28$ weeks in this study (74\%) was higher than that reported among pregnant women in Portugal at early pregnancy (38\%) [31], but lower than that in pregnant women in Scotland at late pregnancy (90\%) [29]. This is consistent with the notion that ferritin concentrations decline with the progression of pregnancy $[32,33]$. These variations may simply reflect normal pregnancy physiology, rather than differences among populations. Though the proportion of our study women with severe iron depletion (ferritin < $15 \mu \mathrm{g} / \mathrm{L}$; $7 \%)$ was much lower than rates reported among pregnant women in other developed countries [10], a high proportion of our women had modest iron depletion (ferritin 15 to $<30 \mu \mathrm{g} / \mathrm{L}$ ). This high number warrants further investigation for subsequent biochemical and clinical consequences among both the mother and offspring.

We found that one in five women reported having been anaemic in the first trimester, similar to the prevalence of anaemia $(22.2 \%)$ in women of reproductive age in Singapore [11]. The vast majority of these women (91\%) were found to be iron deficient at 26-28 weeks despite reported iron-containing supplementation (72\%). Since nearly $80 \%$ of anaemia in the Singapore pregnant population is due to iron deficiency [26], it is likely that most anaemic women in our study already had iron deficiency anaemia in early pregnancy, and even prior to conception. In view that anaemia is a late manifestation of iron deficiency, our findings are not surprising. Of interest is that almost just as high a proportion (75\%) of non-anaemic women in early pregnancy was also found subsequently to be iron deficient at 26-28 weeks. Evaluation of mean corpuscular volume (MCV) and mean corpuscular haemoglobin $(\mathrm{MCH})$ together with ferritin may be required in addition to Hb levels in the screening of women at the beginning of pregnancy to better detect iron deficiency and initiate appropriate iron treatment. Assessment of thalassemia, estimated at 9\% in this population [34], should be considered during the screening procedure as the disorder might lead to abnormalities in the levels of red blood cell indices and biomarkers for iron status. Meanwhile, the role of prophylactic iron supplementation before the onset of iron deficiency anaemia remains controversial, given the reported associations of universal iron supplementation with development of gestational diabetes [35] and pregnancy-induced hypertension [36].

Similar to a local Singapore study in non-pregnant women [37], Malay and Indian pregnant women were more likely to have iron deficiency than Chinese pregnant women. Differences in dietary practice and/ or iron absorption ability may contribute to the higher proportions of iron deficiency in Malays and Indians [37]. Among the ethnic groups, Chinese women have been reported to consume more meat, poultry and egg, contributing to a rich source of haem-iron with a higher bioavailability; Malay women consumed fewer fruits and vegetables and had a lower vitamin $\mathrm{C}$ intake, which could reduce iron absorption; while Indian women tended to be vegetarian where the absorption of non-haem might be inhibited by phytates present in vegetables and cereals $[37,38]$. Younger age may reflect poorer nutrition with reduced dietary iron intake, while multiparity may reflect depleted iron supply with increasing number of pregnancies [28, 32]. The finding of a positive association between education level and iron deficiency is supported by previous reports [27, 39], suggesting that knowledge, understanding or awareness of iron deficiency does not translate into action to increase iron-rich food intake or the use of iron supplements. Despite a high reported use of iron-containing supplements among our pregnant women (88\%), a large proportion of them had iron deficiency at 2628 weeks. The frequency and dosage consumed and compliance with iron-containing supplement intake were not recorded, moreover, which have been reported to influence iron status [10]. Otherwise, the absorption of iron from these iron-containing tablets is probably low due to the absorptive interaction of iron with other divalent metal ions in the tablets (e.g. zinc, manganese, calcium) [40].

We acknowledge several limitations. Variability in plasma volume expansion can affect the interpretation of biomarker levels, including levels of plasma ferritin, particularly late in pregnancy. The best time to detect maternal iron deficiency has been suggested to be in early pregnancy, before the plasma volume is fully expanded. As we did not measure plasma ferritin in early pregnancy, the present findings should therefore be interpreted cautiously. However, the use of a plasma ferritin cut-off of $<30 \mu \mathrm{g} / \mathrm{L}$ to define iron deficiency in the late second trimester is supported by van den Broek and colleagues [18], using bone marrow iron as a reference among pregnant women at mid and late pregnancy. Notwithstanding this, our findings probably include a mix of both physiologic plasma volume expansion and true iron deficiency. Multiple 
indicators of iron status such as transferrin saturation and hepcidin could be collectively used for better determination of iron status at later stages of pregnancy. Data on iron-containing supplementation was self-reported and therefore subject to recall errors. Data on dietary factors that may affect iron absorption were not available in this study. Measurement of $\mathrm{Hb}$ level at 26-28 weeks' gestation is not part of routine clinical assessment in this setting, so data on maternal anaemia status was not available at the same time point as the plasma ferritin measurement. However, the use of a plasma ferritin cut-off of $<15 \mu \mathrm{g} / \mathrm{L}$ has been documented as an indicator for iron deficiency anaemia [17]. The proportions of women who reported smoking and a history of anaemia were small $(\leq 5 \%)$ and insufficient to provide reliable conclusions regarding these groups. Some differences in characteristics (i.e., age and education) were noted between included and excluded women, which might have introduced selection bias. Finally, the lack of data on maternal inflammatory biomarkers (e.g. C-reactive protein and $\alpha$-1-acid glycoprotein) is another limitation. Though sTfR has previously thought to be less affected by inflammation, a recent study found that sTfR has a weak but consistent relation with $\alpha$-1-acid glycoprotein concentrations [41]. Ferritin concentrations are widely recognised to increase in the presence of inflammation [16] and pregnancy has been associated with low-grade inflammation [42]; thus the detection rate of iron deficiency in this study may be underestimated with no adjustment for inflammatory markers [43].

\section{Conclusions}

In conclusion, a substantial proportion of Asian pregnant women were found to be iron deficient at 26-28 weeks in Singapore, highlighting the potential importance of routine monitoring and screening for iron deficiency at several time points during pregnancy for timely commencement of iron treatment. Concerted efforts, including routine dietary advice (e.g. consuming plenty of iron-rich foods with a higher iron bioavailability, along with items containing vitamin C) and individual iron supplementation prophylaxis before and during pregnancy should be considered in this population for optimal maternal and offspring health. However, more research is needed to determine the appropriate use and dose of iron supplements for differing degrees of iron deficiency in pregnancy. Although the treatment of iron deficiency anaemia is not disputed, the maternal and offspring benefits of iron supplementation in iron deficiency with the absence of anaemia require rigorous evaluation in randomized trials.

\section{Additional files}

Additional file 1: Table S1. Comparison of characteristics between excluded and included participants ( $n=1152)$. (DOC $63 \mathrm{~kb}$ )

Additional file 2: Table S2. Factors associated with iron status during pregnancy assessed by ordinal logistic regression analyses $(n=871)$ (DOC 63 kb)

\section{Abbreviations}

BMI: Body mass index; ELISA: Enzyme-linked immunosorbent assay; GUSTO: Growing Up in Singapore Towards healthy Outcomes; Hb: Haemoglobin; KKH: KK Women's and Children's Hospital; MCH: Mean corpuscular haemoglobin; MCV: Mean corpuscular volume; NUH: National University Hospital; sTfR: Soluble transferrin receptor; WHO: World Health Organization

\section{Acknowledgments}

The authors would like to thank the study participants and the GUSTO study group, which includes Allan Sheppard, Amutha Chinnadurai, Anne Eng Neo Goh, Anne Rifkin-Graboi, Anqi Qiu, Arijit Biswas, Bee Wah Lee, Birit F.P. Broekman, Boon Long Quah, Borys Shuter, Chai Kiat Chng, Cheryl Ngo, Choon Looi Bong, Christiani Jeyakumar Henry, Cornelia Yin Ing Chee, Yam Thiam Daniel Goh, Doris Fok, George Seow Heong Yeo, Helen Chen, Hugo P S van Bever, Iliana Magiati, Inez Bik Yun Wong, Ivy Yee-Man Lau, Jeevesh Kapur, Jenny L. Richmond, Joanna D. Holbrook, Joshua J. Gooley, Kenneth Kwek, Krishnamoorthy Niduvaje, Leher Singh, Lin Lin Su, Lourdes Mary Daniel, Marielle V. Fortier, Mark Hanson, Mary Rauff, Mei Chien Chua, Michael Meaney, Mya Thway Tint, Neerja Karnani, Ngee Lek, Oon Hoe Teoh, P. C. Wong, Peter D. Gluckman, Pratibha Agarwal, Rob M. van Dam, Salome A. Rebello, Seang-Mei Saw, Shang Chee Chong, Shirong Cai, Shu-E Soh, Sok Bee Lim, Chin-Ying Stephen Hsu, Victor Samuel Rajadurai, Walter Stunkel, Wee Meng Han, Wei Wei Pang, Yin Bun Cheung, Yiong Huak Chan and Yung Seng Lee.

\section{Funding}

This research was supported by the Singapore National Research Foundation under its Translational and Clinical Research (TCR) Flagship Programme and administered by the Singapore Ministry of Health's National Medical Research Council (NMRC), Singapore- NMRC/TCR/004-NUS/2008; NMRC/TCR/ 012-NUHS/2014. Additional funding was provided by the Singapore Institute for Clinical Sciences, Agency for Science Technology and Research (A*STAR), Singapore. JKYC received salary support from the Ministry of Health's National Medical Research Council, Singapore (NMRC/CSA(SI)/008/2016). KMG is supported by the National Institute for Health Research through the NIHR Southampton Biomedical Research Centre and by the European Union's Seventh Framework Programme (FP7/2007-2013), project EarlyNutrition under grant agreement $n^{\circ} 289346$. None of the funding bodies identified had any role in the design of the study, in data collection, or in analysis, interpretation or writing the manuscript.

\section{Availability of data and materials}

The datasets generated and/or analysed during the current study are not publicly available due to an ethical restriction (patient confidentiality) but are available from the corresponding author on reasonable request.

\section{Authors' contributions}

FY, KMG, LPCS and YSC designed the GUSTO study. SLL, YSC and CC designed the present study. PLQ and MFFC were responsible for the analysis of laboratory samples. MSK provided advice on statistical strategy and analysis. SLL, LML and PTT performed data management and statistical analysis. SLL and LML drafted the paper. SLL, SYC, YLC, JKYC, KHT, KMG, MFFC, MSK, YSC and CC interpreted the findings. All authors participated in the critical review, revision and approval of the final manuscript.

\section{Ethics approval and consent to participate}

Ethical approval was obtained from the Domain Specific Review Board of Singapore National Healthcare Group (reference D/09/021) and the Centralised Institute Review Board of SingHealth (reference 2009/280/D). Informed written consent was obtained from all participants. 


\section{Consent for publication}

Not applicable.

\section{Competing interests}

FY, KMG and YSC have received reimbursement for speaking at conferences sponsored by companies selling nutritional products. SYC, KMG and YSC are part of an academic consortium that has received research funding from Abbott Nutrition, Nestle and Danone. No other disclosures were reported.

\section{Publisher's Note}

Springer Nature remains neutral with regard to jurisdictional claims in published maps and institutional affiliations.

\section{Author details}

'Department of Reproductive Medicine, KK Women's and Children's Hospital, 100 Bukit Timah Road, Singapore 229899, Singapore. ${ }^{2}$ Duke-NUS Medical School, 8 College Road, Singapore 169857, Singapore. ${ }^{3}$ Singapore Institute for Clinical Sciences, Agency for Science, Technology and Research, (A*STAR), 30 Medical Drive, Singapore 117609, Singapore. ${ }^{4}$ Department of Obstetrics \& Gynaecology, National University Hospital, 5 Lower Kent Ridge Road, Singapore 119074, Singapore. ${ }^{5}$ Department of Obstetrics \& Gynaecology, Yong Loo Lin School of Medicine, National University of Singapore, National University Health System, 1E Kent Ridge Road, Singapore 119228, Singapore. ${ }^{6}$ Department of Haematology-Oncology, National University Cancer Institute, NUH Medical Centre, 5 Lower Kent Ridge Road, Singapore 119074, Singapore. ${ }^{7}$ Department of Maternal Fetal Medicine, KK Women's and Children's Hospital, 100 Bukit Timah Road, Singapore 229899, Singapore. ${ }^{8}$ Department of Paediatrics, KK Women's and Children's Hospital, 100 Bukit Timah Road, Singapore 229899, Singapore. ${ }^{\circ}$ Lee Kong Chian School of Medicine, Nanyang Technological University, 11 Mandalay Road, Singapore 308232, Singapore. ${ }^{10}$ Medical Research Council Lifecourse Epidemiology Unit, University of Southampton, Southampton SO16 6YD, UK. ${ }^{11}$ National Institute for Health Research Southampton Biomedical Research Centre, University of Southampton and University Hospital Southampton National Health Service Foundation Trust, Southampton SO16 6YD, UK. ${ }^{12}$ Department of Paediatrics, Yong Loo Lin School of Medicine, National University of Singapore, 1E Kent Ridge Road, NUHS Tower Block Level 12, Singapore 119228, Singapore.

${ }^{13}$ Khoo Teck Puat-National University Children's Medical Institute, National University Hospital, National University Health System, 5 Lower Kent Ridge Road, Singapore 119074, Singapore. ${ }^{14}$ Saw Swee Hock School of Public Health, National University of Singapore, 12 Science Drive 2, Singapore 117549, Singapore. ${ }^{15}$ Department of Pediatrics, McGill University Faculty of Medicine, Montreal, QC H3G 2M1, Canada. ${ }^{16}$ Department of Epidemiology, Biostatistics and Occupational Health, McGill University Faculty of Medicine, Montreal, QC H3A 1A2, Canada.

\section{Received: 12 November 2018 Accepted: 2 April 2019} Published online: 11 April 2019

\section{References}

1. TO S. Iron status during pregnancy: setting the stage for mother and infant. Am J Clin Nutr. 2005:81:1218S-22S.

2. World Health Organization. Nutrition: Micronutrient deficiencies. https:// www.who.int/nutrition/topics/micronutrients/en/ (2017). Accessed 15 June 2017.

3. Pavord S, Myers B, Robinson S, Allard S, Strong J, Oppenheimer C, et al. UK guidelines on the management of iron deficiency in pregnancy. $\mathrm{Br} J$ Haematol. 2012;156:588-600.

4. Breymann C. Iron deficiency Anemia in pregnancy. Semin Hematol. 2015:52:339-47.

5. World Health Organization. United Nations Children's fund, United Nations University. Iron deficiency anaemia: assessment, prevention, and control: a guide for programme managers. In: Geneva: WHO; 2001.

6. EFSA Panel on Dietetic Products Nutrition and Allergies (NDA). Scientific opinion on dietary reference values for iron. EFSA J. 2015:13:4254.

7. Department of Health. Dietary reference values for food energy and nutrients for the United Kingdom. Report of the panel on dietary reference values of the committee on medical aspects of food policy. London: HMSO; 1991.

8. Williamson CS. Nutrition in pregnancy. Nutr Bull. 2006;31:28-59.
9. Aranda N, Ribot B, Garcia E, Viteri FE, Arija V. Pre-pregnancy iron reserves, iron supplementation during pregnancy, and birth weight. Early Hum Dev. 2011;87:791-7.

10. Milman N, Taylor CL, Merkel J, Brannon PM. Iron status in pregnant women and women of reproductive age in Europe. Am J Clin Nutr. 2017;106:1655S-62S.

11. World Health Organization. Global health observational data repository: prevalence of anaemia in women, estimates by country. http://apps.who. int/gho/data/view.main.GSWCAH28v?lang=en (2016). Accessed 12 Feb 2019.

12. Scholl TO. Maternal iron status: relation to fetal growth, length of gestation and the neonate's iron endowment. Nutr Rev. 2011;69:S23-9.

13. Radlowski EC, Johnson RW. Perinatal iron deficiency and neurocognitive development. Front Hum Neurosci. 2013;7:585.

14. Lubach GR, Coe CL. Preconception maternal iron status is a risk factor for iron deficiency in infant rhesus monkeys (Macaca mulatta). J Nutr. 2006:136:2345-9.

15. Jaime-Perez JC, Herrera-Garza JL, Gomez-Almaguer D. Sub-optimal fetal iron acquisition under a maternal environment. Arch Med Res. 2005;36:598-602.

16. Daru J, Allotey J, Pena-Rosas JP, Khan KS. Serum ferritin thresholds for the diagnosis of iron deficiency in pregnancy: a systematic review. Transfus Med. 2017;27:167-74.

17. American College of Obstetricians and Gynecologists. ACOG Practice bulletin No.95: Anemia in pregnancy. Obstet Gynecol. 2008;112:201-7.

18. van den Broek NR, Letsky EA, White SA, Shenkin A. Iron status in pregnant women: which measurements are valid? Brit J Haematol. 1998;103:817-24.

19. Soh SE, Tint MT, Gluckman PD, Godfrey KM, Rifkin-Graboi A, Chan YH, et al. Cohort profile: growing up in Singapore towards healthy outcomes (GUSTO) birth cohort study. Int J Epidemiol. 2014:43:1401-9.

20. World Health Organization. Appropriate body-mass index for Asian populations and its implications for policy and intervention strategies. Lancet. 2004:363:157-63.

21. Choi JW, Im MW, Pai SH. Serum transferrin receptor concentrations during normal pregnancy. Clin Chem. 2000;46:725-7.

22. Scott SC, Goldberg MS, Mayo NE. Statistical assessment of ordinal outcomes in comparative studies. J Clin Epidemiol. 1997;50:45-55.

23. Sun GW, Shook bTL, Kay GL. Inappropriate use of bivariable analysis to screen risk factors for use in multivariable analysis. J Clin Epidemiol. 1996:49:907-16

24. Masukume G, Khashan AS, Kenny LC, Baker PN, Nelson G, SCOPE Consortium. Risk factors and birth outcomes of anaemia in early pregnancy in a nulliparous cohort. PLoS One. 2015;10:e0122729.

25. Reddy OC, Alemayehu E. Ordinal logistic regression analysis to assess the factors that affect health status of students in Ambo University: a case of natural and computational sciences college. Ambo University. 2015;2:153-63.

26. Singh $\mathrm{K}$, Fong YF, Arulkumaran S. Anaemia in pregnancy--a cross-sectional study in Singapore. Eur J Clin Nutr. 1998;52:65-70.

27. Adams C, Costello A, Flynn S. Iron deficiency anaemia in ecuador: does education matter. 2007. https://pdfs.semanticscholar.org/cf26/ 89806194d67bf893d3615f398dc7e8f096db.pdf. Accessed 5 Feb 2018.

28. Khambalia AZ, Collins CE, Roberts CL, Morris JM, Powell KL, Tasevski V, et al. Iron deficiency in early pregnancy using serum ferritin and soluble transferrin receptor concentrations are associated with pregnancy and birth outcomes. Eur J Clin Nutr. 2015;70:358-63.

29. Shields RC, Caric V, Hair M, Jones O, Wark L, McColl MD, et al. Pregnancyspecific reference ranges for haematological variables in a Scottish population. J Obstet Gynaecol. 2011:31:286-9.

30. Alwan NA, Cade JE, McArdle HJ, Greenwood DC, Hayes HE, Simpson NA. Maternal iron status in early pregnancy and birth outcomes: insights from the Baby's vascular health and Iron in pregnancy study. Br J Nutr. 2015:113:1985-92

31. da Costa AG, Vargas S, Clode N, Graca LM. Prevalence and risk factors for Iron deficiency Anemia and Iron depletion during pregnancy: a prospective study. Acta Medica Port. 2016:29:514-8.

32. Mei Z, Cogswell ME, Looker AC, Pfeiffer CM, Cusick SE, Lacher DA, et al. Assessment of iron status in US pregnant women from the National Health and nutrition examination survey (NHANES), 1999-2006. Am J Clin Nutr. 2011;93:1312-20.

33. Rawal S, Hinkle SN, Bao W, Zhu Y, Grewal J, Albert PS, et al. A longitudinal study of iron status during pregnancy and the risk of gestational diabetes: findings from a prospective, multiracial cohort. Diabetologia. 2017;60:249-57.

34. Kham SK, Quah TC, Loong AM, Tan PL, Fraser A, Chong SS, et al. A molecular epidemiologic study of thalassemia using newborns' cord blood 
in a multiracial Asian population in Singapore: results and recommendations for a population screening program. J Pediatr Hematol Oncol. 2004;26:817-9.

35. Brannon PM, Taylor CL. Iron supplementation during pregnancy and infancy: uncertainties and implications for research and policy. Nutrients. 2017:9:1327.

36. Ouladsahebmadarek E, Sayyah-Melli M, Taghavi S, Abbasalizadeh S, Seyedhejazie M. The effect of supplemental iron elimination on pregnancy outcome. Pak J Med Sci. 2011;27:641-5.

37. Hughes K. Serum ferritin and iron status in the general population of Singapore, 1993 to 1995. Ann Acad Med Singap. 1998;27:507-11.

38. Health Promotion Board. Report of the National Nutrition Survey 2010, Singapore. https://www.hpb.gov.sg/docs/default-source/pdf/nns-2010report.pdf?sfvrsn=18e3f172_2 (2010). Accessed 15 Feb 2019.

39. Schuepbach RA, Bestmann L, Bechir M, Fehr J, Bachli EB. High prevalence of Iron deficiency among educated hospital employees in Switzerland. Int J Biomed Sci. 2011;7:150-7.

40. Milman N. Oral iron prophylaxis in pregnancy: not too little and not too much! J Pregnancy. 2012;2012:514345.

41. Rohner F, Namaste SML, Larson LM, Addo OY, Mei Z, Suchdev PS, et al. Adjusting soluble transferrin receptor concentrations for inflammation: biomarkers reflecting inflammation and nutritional determinants of Anemia (BRINDA) project. Am J Clin Nutr. 2017;106:372S-82S

42. Wang Q, Würtz P, Auro K, Mäkinen VP, Kangas AJ, Soininen P, et al. Metabolic profiling of pregnancy: crosssectional and longitudinal evidence. BMC Med. 2016;14:205.

43. Namaste SML, Rohner F, Huang J, Bhushan NL, Flores-Ayala R, Kupka R, et al. Adjusting ferritin concentrations for inflammation: biomarkers reflecting inflammation and nutritional determinants of Anemia (BRINDA) project. Am J Clin Nutr. 2017;106:359S-71S.

Ready to submit your research? Choose BMC and benefit from:

- fast, convenient online submission

- thorough peer review by experienced researchers in your field

- rapid publication on acceptance

- support for research data, including large and complex data types

- gold Open Access which fosters wider collaboration and increased citations

- maximum visibility for your research: over $100 \mathrm{M}$ website views per year

At BMC, research is always in progress.

Learn more biomedcentral.com/submissions 